\title{
Effect of coculturing canine notochordal, nucleus pulposus and mesenchymal stromal cells for intervertebral disc regeneration
}

Irene TM Arkesteijn ${ }^{1}$, Lucas A Smolders ${ }^{2,3}$, Sandra Spillekom², Frank M Riemers², Esther Potier ${ }^{1,4}$, Björn P Meij ${ }^{2}$, Keita Ito ${ }^{1,5}$ and Marianna A Tryfonidou ${ }^{2^{*}}$

\begin{abstract}
Introduction: Early degenerative changes in the nucleus pulposus (NP) are observed after the disappearance of notochordal cells (NCs). Thus, it has been suggested that NCs play an important role in maintaining the NP and may have a regenerative potential on other cells of the NP. As the number of resident NP cells (NPCs) decreases in a degenerating disc, mesenchymal stromal (stem) cells (MSCs) may be used for cell supplementation. In this study, using cells of one species, the regenerative potential of canine NCs was assessed in long-term three-dimensional coculture with canine NPCs or MSCs.
\end{abstract}

Methods: Canine NCs and canine NPCs or MSCs were cocultured in alginate beads for 28 days under hypoxic and high-osmolarity conditions. Cell viability, cell morphology and DNA content, extracellular matrix production and expression of genes related to NC markers (Brachyury, KRT18) and NP matrix production (ACAN, COL2A1, COL1A1) were assessed after 1, 15 and 28 days of culture.

Results: NCs did not completely maintain their phenotype (morphology, matrix production, gene expression) during 28 days of culture. In cocultures of NPCs and NCs, both extracellular matrix content and anabolic gene expression remained unchanged compared with monoculture groups, whereas cocultures of MSCs and NCs showed increased glycosaminoglycan/DNA. However, the deposition of these proteoglycans was observed near the NCs and not the MSCs. Brachyury expression in the MSC and NC coculture group increased in time. The latter two findings indicate a trophic effect of MSCs on NCs rather than vice versa.

Conclusions: No regenerative potential of canine NCs on canine NPCs or MSCs was observed in this study. However, significant changes in NC phenotype in long-term culture may have resulted in a suboptimal regenerative potential of these NCs. In this respect, NC-conditioned medium may be better than coculture for future studies of the regenerative potential of NCS.

\section{Introduction}

Low back pain is the most common cause of disability worldwide; in many cases, it is attributable to intervertebral disc (IVD) degeneration [1]. Current therapies for low back pain are symptom-oriented and are successful in relieving pain. However, they do not preserve the function of the IVD, and, in the long term, the results of treatment are suboptimal. Because the first signs of disc

\footnotetext{
* Correspondence: m.a.tryfonidou@uu.nl

${ }^{2}$ Department of Clinical Sciences of Companion Animals, Faculty of Veterinary Medicine, Utrecht University, PO Box 80.154, NL-3508 TD Utrecht, The Netherlands

Full list of author information is available at the end of the article
}

degeneration are observed in the core of the disc, called the nucleus pulposus (NP), regeneration of the NP is of great interest for designing new therapies to maintain the function of the IVD [2].

Degeneration of the IVD involves the transition from a gelatinous to a fibrotic NP [3]. This change is associated with a decreased ability of the NP to convert compressive forces into evenly distributed tensile stresses in the surrounding annulus fibrosus (AF), with consequent degeneration of the AF [4]. Both the resident cells and extracellular matrix of the NP undergo major changes in this degenerative process. At birth, a human NP is populated by clusters of large, vacuolated notochordal cells (NCs) and by small, 
chondrocyte-like nucleus pulposus cells (NPCs), whereas a degenerating NP is populated by increasingly apoptotic NPCs and possibly fibrochondrocyte-like cells [5]. Furthermore, the healthy gelatinous NP is rich in proteoglycans that keep the tissue hydrated, whereas in the degenerated NP, matrix contains less proteoglycans, different collagen types and more matrix-degrading enzymes [5].

Interestingly, degenerative changes are observed after loss of NCs. Similarly to humans, chondrodystrophic dog breeds show loss of NCs early in life. These dogs develop generalized IVD degeneration as young adults and are predisposed to develop subsequent IVD diseases, such as herniation, later in life [6-8]. Conversely, nonchondrodystrophic dog breeds maintain gelatinous NPs, rich in NCs, far into adulthood and generally develop IVD diseases only at an advanced age and in isolated locations in the spine [9]. Therefore, it has been suggested that NCs play an important role in maintaining the NP by synthesizing new matrix [10] and by regulating matrix synthesis of other cells [11]. Because of the fact that NPC + NC cocultures and cultures of NPCs in NCconditioned medium have resulted in a significant increase in proteoglycan production by NPCs [11-13], $\mathrm{NCs}$ are interesting targets for research strategies to regenerate the NP.

As the number of NPCs decreases in a degenerating disc, it may also be necessary to complement the resident cell population to maintain the health of the NP matrix. Repopulation of the degenerating disc with healthy NPCs would be a logical strategy. However, harvesting NPCs is likely to induce degeneration of donor discs. Alternatively, autologous mesenchymal stromal (stem) cells (MSCs) are readily available, show high proliferation rates and can differentiate into NPC-like cells $[14,15]$. MSC cultures in NC-conditioned medium have resulted in increased proteoglycan synthesis [16], and, when injected into NPs, MSCs showed increased matrix production and proliferation $[17,18]$.

It is known that NCs, MSCs and NPCs cocultured in different combinations have a certain regenerative potential $[11,13]$; however, owing to varying culture conditions, previous studies have not allowed a valid comparison of the regenerative potential of NCs cocultured with NPCs with NCs cocultured with MSCs. Therefore, in this article, we present the first long-term study of coculture of canine NCs with NPCs or MSCs, within the same species. Articular chondrocytes, with a phenotype similar to but ECM production different from NPCs [19], were simultaneously cocultured with NPCs or MSCs to assess NC-specific effects in coculture. We hypothesized that canine NCs stimulate the production of extracellular matrix by canine NPCs and MSCs when cultured in three dimensions and under near-physiological NP conditions (approximately native cell density, hypoxia and adjusted osmolarity). To evaluate the regenerative effect of NCs on NPCs and MSCs in coculture, we compared the effect of NCs on (1) MSCs, with articular chondrocytes (ACs) as a control group; (2) NPCs, with ACs as a control group; and (3) NPCs, with MSCs as a control group.

\section{Methods}

\section{Cell isolation and expansion}

All cells used in this study were obtained from healthy dogs killed for unrelated experiments, which were approved by the ethics committee on animal experimentation of Utrecht University (DEC: 2012.III.05.046). Experiments were conducted with freshly isolated NCs and cryopreserved, bone marrow-derived MSCs, NPCs and ACs.

Complete spines (cervical, thoracic and lumbosacral regions) were collected from four mongrel dogs (nonchondrodystrophic (NCD1 through NCD4); female, age $1.3 \pm 0.5$ years, weight $26.0 \pm 14.6 \mathrm{~kg}$ (mean \pm standard deviation (SD)). The technique of harvesting NPs has been developed by experienced, board-certified veterinary surgeons (BM and MAT) who are familiar with IVD-related spine surgery in canine patients with IVD diseases. The technique was optimized to expose and retrieve 26 discs (6 cervical, 13 thoracolumbar and 7 lumbar IVDs) within the time span of 1 hour in a canine cadaveric spine. Care was taken to include only NP tissue and not AF or endplate tissue. NP tissue was pooled per donor in Dulbecco's modified Eagle's medium (DMEM)/Ham's F-12 (GlutaMAX; Life Technologies, Carlsbad, CA, USA) $+2 \%$ penicillin/streptomycin (P/S; PAA Laboratories, Cölbe, Germany). The pooled NPs were digested according to the method used by Smolders et al. [20]: 0.1\% pronase (Roche Diagnostics, Almere, The Netherlands) for $45 \mathrm{mi}-$ nutes and $0.05 \%$ collagenase type II (Worthington Biochemical, Lakewood, NJ, USA) overnight, both at $37^{\circ} \mathrm{C}$. Subsequently, the cell suspension was filtered with a 40$\mu \mathrm{m}$ cell strainer (BD Biosciences, Erembodegem, Belgium), and cells $>40 \mu \mathrm{m}$ (mostly clusters) were flushed away from the strainer surface with culture medium and collected. After centrifugation (at $500 \mathrm{~g}$ for 5 minutes at room temperature), cell cluster pellets were resuspended in $100 \%$ fetal bovine serum (FBS) (FBS Gold; PAA Laboratories). Per dog, $26.0 \pm 12.3 \times 10^{6}$ (mean \pm SD) living cells were counted in a propidium iodide (PI) assay (Nucleocounter NC-100; Chemometec, Nieuwegein, The Netherlands). Fluorescent PI can bind double-stranded DNA but is unable to permeate the membrane of living cells. In this assay, the number of viable cells was determined by calculating the difference between the number of dead cells in suspension before (dead cell concentration) and after lysis of the cell membranes (total cell concentration, including clustered cells).

MSCs, NPCs and ACs were harvested from eight Beagle dogs (chondrodystrophic (CD1 through CD8; male, age 
$2.0 \pm 0.3$ years, weight $12.0 \pm 1.3 \mathrm{~kg}($ mean $\pm \mathrm{SD}))$. For each donor, bone marrow was collected and MSCs were isolated as described elsewhere [21]. When $80 \%$ confluence was reached (within 7 days), MSCs were cryopreserved at P0.

Cervical and thoracic spines were collected, and NPs were harvested and pooled per donor as described above for NC isolation. ACs were obtained from both stifle joints. After the joint was opened, cartilage was harvested from the distal femoral condyles, the patella and the proximal tibial plateau. NPs and knee cartilage were digested in $0.15 \%$ pronase for 45 minutes and $0.15 \%$ collagenase type II overnight, both at $37^{\circ} \mathrm{C}$. The cell suspension was filtered with a $70-\mu \mathrm{m}$ cell strainer (BD Biosciences), and the NPCs and ACs were collected from the filtrate by centrifugation. The yield per dog was $7.0 \pm 3.0 \times 10^{6}$ living NPCs and $14.2 \pm 3.6 \times 10^{6}$ living ACs (mean $\pm \mathrm{SD}$ ). The cells were cryopreserved directly after isolation (P0). MSCs, NPCs and ACs were thawed and expanded 6 days before the isolation of NCs. MSCs were cultured up to passage 2, whereas NPCs and ACs were cultured up to passage 1 . All three cell types were cultured in high-glucose (4.5 g/L) DMEM (Life Technologies) $+10 \%$ FBS (Greiner Bio-One, Alphen aan den Rijn, The Netherlands) + 1\% P/S (Lonza, Basel, Switzerland).

\section{Experimental design}

To compare the stimulation potential of NCs, NCs were cocultured with MSCs or NPCs separately. In order to identify whether the observed effects were $\mathrm{NC}$-specific, ACs were used in place of NCs in the same combinations. Monoculture controls for each individual cell type were also conducted. Finally, the effect of MSCs on NPCs in coculture was also examined (Table 1). For each experiment repetition, multiple MSC, NPC and AC donors were pooled, and different combinations of MSCs, NPCs and ACs were used for each NC donor (Table 2).

$\begin{aligned} & \text { Table } \mathbf{1} \text { Experimental groups, cell combinations, cell } \\
& \text { concentrations and } \text { repeats }^{\mathbf{a}}\end{aligned}$
\begin{tabular}{llll} 
Group & Cell types & $\begin{array}{l}\text { Cell concentration } \\
\left(\times 10^{6} / \mathbf{m l} \text { alginate }\right)\end{array}$ & $\begin{array}{l}\text { Number of repeat } \\
\text { experiments }\end{array}$ \\
\hline 1 & NC & 3 & $3(4$ for day 1$)$ \\
2 & MSC & 3 & 4 \\
3 & NPC & 3 & 4 \\
4 & AC & 3 & 4 \\
5 & MSC + NC & $3+3$ & $3(4$ for day 1$)$ \\
6 & NPC + NC & $3+3$ & $3(4$ for day 1$)$ \\
7 & NPC + MSC & $3+3$ & 2 \\
8 & MSC + AC & $3+3$ & 4 \\
9 & NPC + AC & $3+3$ & 3 \\
\hline
\end{tabular}

${ }^{a} \mathrm{NC}$, Notochordal cell; MSC, Mesenchymal stromal cell; NPC, Nucleus pulposus cell; AC, Articular chondrocyte.
Table 2 Cell pooling for each experimental repetition ${ }^{a}$

\begin{tabular}{lllll}
\hline Repetition & NC donor & MSC donors & NPC donors & AC donors \\
\hline 1 & NCD1 & $C D 2,4,8$ & $C D 2,5$ & $C D 2,4$ \\
2 & NCD2 & $C D 2,8$ & $C D 6,7,8$ & $C D 2,4$ \\
3 & $N C D 3$ & $C D 1,2$ & $C D 3,4$ & $C D 4,5$ \\
4 & NCD4 & $C D 3,8$ & $C D 6,7,8$ & $C D 2,4$ \\
\hline
\end{tabular}

${ }^{\mathrm{a} N C}$, Notochordal cell; MSC, Mesenchymal stromal cell; NPC, Nucleus pulposus cell; AC, Articular chondrocyte; CD, Chondrodystrophic; NCD, Nonchondrodystrophic.

The number of repetitions for each cell group is shown in Table 1. Alginate beads of these cell combinations were made as previously described for semisolid beads by Guo et al. [22] using a 26-gauge needle and $1.2 \% \mathrm{w} / \mathrm{v}$ alginate (Sigma-Aldrich, St Louis, MO, USA). The cell concentration for monocultures was $3 \times 10^{6} / \mathrm{ml}$ alginate and $6 \times 10^{6} / \mathrm{ml}$ alginate for cocultures (Table 1). The cell concentration was doubled in cocultures to assess the effect of the regulatory cells. The alginate beads were cultured in agarose-coated (to avoid cell adherence) well plates at $37^{\circ} \mathrm{C}, 5 \% \mathrm{CO}_{2}$ and $5 \% \mathrm{O}_{2}$ (hypoxia) for 28 days. The medium consisted of high-glucose DMEM $+5 \%$ $\mathrm{FBS}+1 \%$ sodium pyruvate (Lonza) $+1 \% \mathrm{P} / \mathrm{S}+1 \% 0.4 \mathrm{M}$ $\mathrm{KCl}$ (Merck, Darmstadt, Germany) $+1 \% 5 \mathrm{M} \mathrm{NaCl}$ (Merck) and was changed twice weekly. The latter two medium components were used to adjust the osmolarity of the medium to $400 \mathrm{mOsm} / \mathrm{L}$, similar to the osmolarity in healthy bovine NP tissue $[23,24]$.

\section{Assessments}

Samples were analyzed on days 1,15 and 28 for cell viability and morphology, proteoglycan production and gene expression.

To assess cell viability, alginate beads were incubated for 1 hour in $10 \mu \mathrm{M}$ calcein-AM and $10 \mu \mathrm{M}$ PI (both from Molecular Probes, Eugene, OR, USA) in phosphatebuffered saline. Cells were imaged using a confocal microscope (CLSM 510 META NLO; Carl Zeiss, Sliedrecht, The Netherlands). The beads were assessed at a depth range of $50 \mu \mathrm{m}$ to $200 \mu \mathrm{m}$ from the bead surface.

To assess cell morphology and matrix deposition, alginate beads were fixed in 3.7\% formalin (Merck) +100 $\mathrm{mM} \mathrm{CaCl}{ }_{2}$ (Calbiochem, Darmstadt, Germany). After ethanol dehydration, the beads were embedded in paraffin. Subsequently, $5-\mu \mathrm{m}$-thick paraffin sections were cut (RM2255; Leica, Rijswijk, The Netherlands) and stained with hematoxylin and eosin (cell morphology) or Safranin $\mathrm{O} /$ Fast Green/hematoxylin to assess matrix deposition. The sections were examined by light microscopy (BX60; Olympus, Zoeterwoude, The Netherlands) and a color charge-coupled device camera (Leica).

Three alginate beads per time point were digested overnight at $60^{\circ} \mathrm{C}$ in $450 \mu \mathrm{l}$ of papain digestion buffer (100 $\mathrm{mM}$ phosphate buffer; $5 \mathrm{mM}$ ethylenediaminetetraacetic 
acid, disodium dihydrate; $5 \mathrm{mM}$ L-cysteine hydrochloride, anhydrous; and 125 to $140 \mu \mathrm{g} / \mathrm{ml}$ papain (all from Sigma-Aldrich) at $\mathrm{pH}$ 6.0) for biochemical analysis. The DNA content was measured with a Hoechst dye assay [25] using calf thymus DNA (Sigma-Aldrich) as a reference. With the same solution, the sulfated glycosaminoglycan (GAG) content was measured using a modified dimethylmethylene blue assay [26] ( $\mathrm{pH}$ 1.5) using shark cartilage chondroitin sulfate (Sigma-Aldrich) as a reference. GAG values were normalized to the DNA content (GAG/DNA). The DNA was expressed per alginate bead. The hydroxyproline (HYP) content, as a measure for collagen, was determined in a chloramine-T assay [27] using trans-4-hydroxyproline (Sigma-Aldrich) as a reference. The HYP content was below detection limits in all samples.

Cells were isolated from five alginate beads per group by a 5 -minute incubation in sodium citrate digestion buffer $(55 \mathrm{mM}$ sodium citrate, Sigma-Aldrich; $0.15 \mathrm{M}$ $\mathrm{NaCl}$, Merck; $25 \mathrm{mM}$ 4-(2-hydroxyethyl)piperazine-1ethanesulfonic acid, Sigma-Aldrich) for gene expression analysis. Subsequently, cells were lysed in $300 \mu \mathrm{l}$ of Buffer RLT (RNeasy Mini Kit; Qiagen, Leusden, The Netherlands) $+1 \% \quad \beta$-mercaptoethanol (Sigma-Aldrich) and stored at $-80^{\circ} \mathrm{C}$ until RNA isolation. Total RNA was isolated using the RNeasy Micro Kit (Qiagen) according to the manufacturer's instructions. The quantity and purity of RNA were determined with a spectrophotometer (NanoDrop ND-1000; Thermo Fisher Scientific, Wilmington, DE, USA). Subsequently, the iScript cDNA Synthesis Kit (Bio-Rad Laboratories, Veenendaal, The Netherlands) was used to reverse-transcribe the total RNA. Gene expression was analyzed in a quantitative RT-PCR (qPCR) (CFX384, Bio-Rad Laboratories) experiment using iQ SYBR Green Supermix (Bio-Rad Laboratories). Relative quantification was calculated using the comparative threshold cycle $\left(2^{-\Delta \Delta C t}\right)$ algorithm with kinetic PCR efficiency correction [28], with the results normalized to the reference gene and day 1 of the same gene. Relative gene expression was measured for the reference genes ribosomal protein S19 (RPS19), TATA boxbinding protein $(T B P)$ and glyceraldehyde 3-phosphate dehydrogenase $(G A P D H)$, and the target genes: (1) NC markers brachyury and cytokeratin 18 (KRT18) [19] and (2) matrix production-associated genes aggrecan $(A C A N)$; collagen, type I, $\alpha 1$ (COL1A1); collagen, type II, $\alpha 1$ (COL2A1); primer sequences (Additional file 1).

\section{Data analysis and statistics}

Statistical analyses were performed using $\mathrm{R}$ statistical software [29]. Linear mixed models [30], containing both fixed (culture duration and cell group) and random effects (individual dog), were used to analyze the described parameters separately for the qPCR and GAG/DNA analyses. A random intercept for each dog was added to each model to take the correlation of the observations within a dog into account. The Akaike Information Criterion was used for model selection. If necessary, models were optimized by correcting for unequal variances and/or for autoregressive correlation. Conditions for the use of mixed models, including normal distribution of the data, were assessed by analyzing the residuals (probability-probability and quantile-quantile plots) of the acquired models. No violations of these conditions were observed.

In order to compare increases in DNA content between culture groups and time points, differences in DNA content between different time points in culture were calculated for all groups and expressed as percentage changes. These values were used as readout parameters in the above-described models.

Because cell culture groups were composed of different cell types and cell combinations, GAG production was normalized by cell number (calculated as GAG/ DNA ratio). Using this parameter, the GAG production between different time points was compared within all culture groups. Also, GAG/DNA values were normalized to values obtained at day 1 in order to compare different cell culture groups in their increase or decrease in time. These values were used as readout parameters in the statistical models as described above.

For the gene expression analysis, we first evaluated whether the expression of genes of interest differed significantly between different groups on day 1 in culture. For this purpose, the $\Delta C_{\mathrm{t}}$ value for individual target genes at day 1 in culture was used. To evaluate the effect of combing two cell types on the gene expression of relevant genes, the change for each culture group relative to its own expression on day 1 (as different culture groups showed significant differences in baseline gene expression on day 1) was statistically assessed using the parameter $\Delta \Delta C_{\mathrm{t}} . \Delta \Delta C_{\mathrm{t}}$ was calculated for both days 15 and 28 in culture and was defined as $\left(\Delta C_{\mathrm{t}}\right.$ (day 15) $-\Delta C_{\mathrm{t}}$ (day 1$))$ and $\left(\Delta C_{\mathrm{t}}\right.$ (day 28) $-\Delta C_{\mathrm{t}}$ (day 1$\left.)\right)$ for days 15 and 28 in culture, respectively.

With the aim of answering the research questions, $P$ values were calculated for the above-described parameters for the following comparisons:

- To assess the effect of NCs on MSCs, with ACs as control group: Groups compared were NC, MSC, $\mathrm{AC}, \mathrm{MSC}+\mathrm{NC}$, and MSC + AC.

- To assess the effect of NCs on NPCs, with ACs as control group: Groups compared were NC, NPC, $\mathrm{AC}, \mathrm{NPC}+\mathrm{NC}$, and NPC + AC.

- To assess the effect of NCs on NPCs, MSCs as control group: Groups compared were NC, MSC, $\mathrm{NPC}, \mathrm{MSC}+\mathrm{NC}, \mathrm{NPC}+\mathrm{NC}$, and MSC + NPC. 
For all the described models, the Benjamini-Hochberg method [31] was used to correct for multiple comparisons. $P<0.05$ was considered statistically significant.

\section{Results}

\section{Notochordal cells in monoculture}

On day 1, large, vacuolated NCs were primarily organized in clusters (Figure 1A). The size of the vacuoles and the size of $\mathrm{NC}$ clusters decreased with time, especially during the first 2 weeks. At day 28, three NC morphological phenotypes could be observed: (1) in clusters, with smaller vacuoles than on day 1 ; (2) in clusters of fibroblast-like cells; and (3) occasionally in small clusters of round cells (Figure 1B,C and D, respectively).

On day 28, more GAG was found surrounding the vacuolated NCs than the fibroblast-like NCs (NC row in Figure 2). NC viability was high on day 1 (Additional file 2). GAG/DNA increased significantly with time (Figure 1F, Additional file 3), consistent with Safranin $\mathrm{O} /$ Fast Green staining. The gene expression of $A C A N$ decreased significantly on day 15 , but thereafter it returned to values found at day 1 of culture. The expression of both COL2A1 and COL1A1 increased significantly over time (Figure 1H,I,J, respectively, and
Additional file 4). Furthermore, the expression of NC markers brachyury and KRT18 remained stable over 28 days (Figure 1G, Additional files 4 and 5).

\section{The regulatory effect of notochordal cells on} mesenchymal stromal (stem) cells in coculture

On day 28, morphologies of cocultured NCs, MSCs and ACs were the same as each individual cell type in monoculture (Additional file 6). The cell viability was high on day 1 (Additional file 2) and the DNA content within all culture groups remained statistically unchanged over time (Figure 3, Additional file 3).

In each group, the amount of GAG/DNA increased significantly with time, with the MSC + AC group showing a significantly higher increase than the MSC group (Additional file 3). This was in line with the Safranin O staining, which showed that MSCs + ACs produced more proteoglycans than MSCs alone (Figure 2). Although not statistically significant, the average GAG/DNA in the $\mathrm{MSC}+\mathrm{NC}$ group on day 28 was notably higher compared with the MSCs and NCs monoculture groups. This effect was also seen in the Safranin O staining, which showed that the NCs produced more proteoglycans in the presence of MSCs (Figure 2).

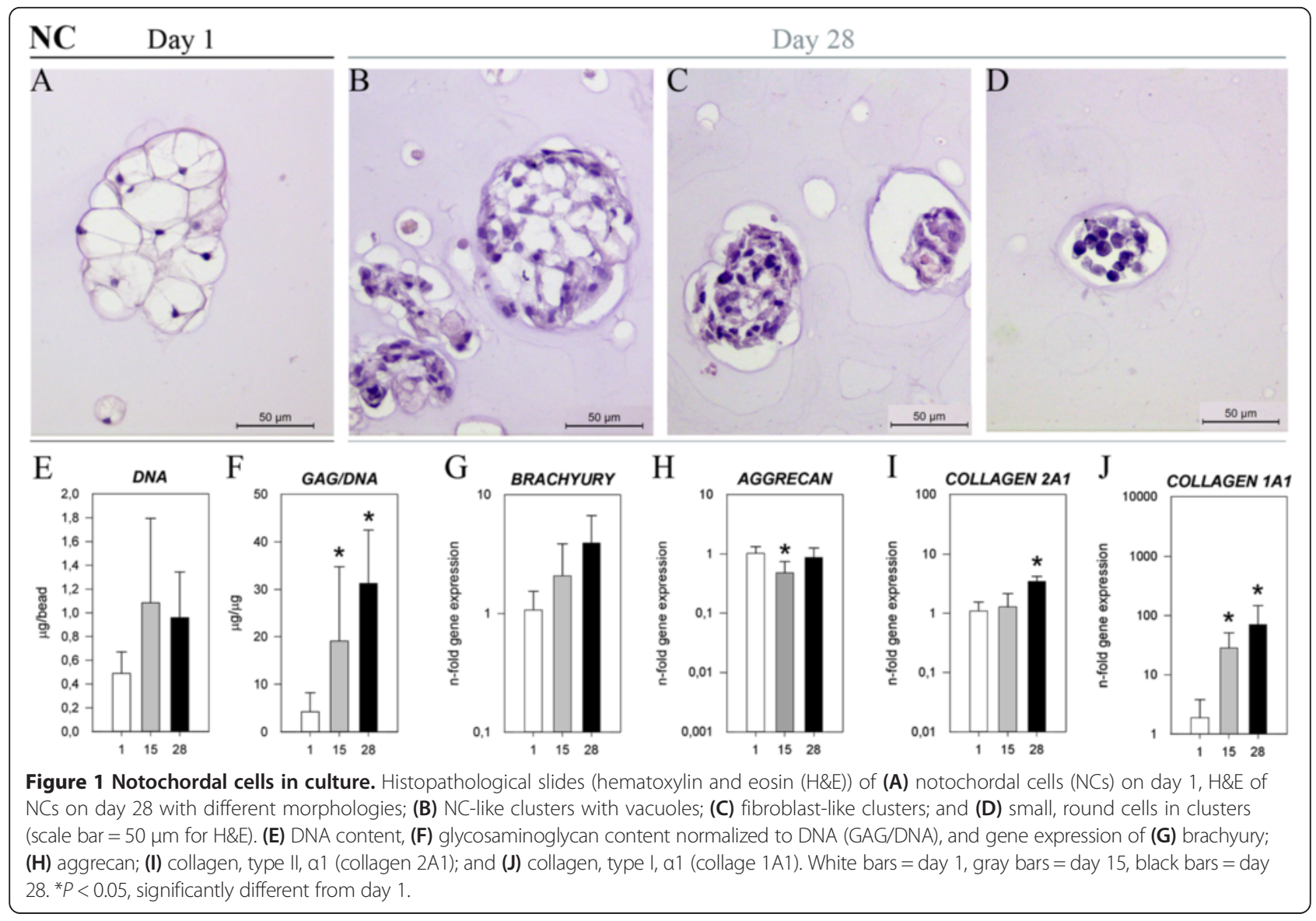




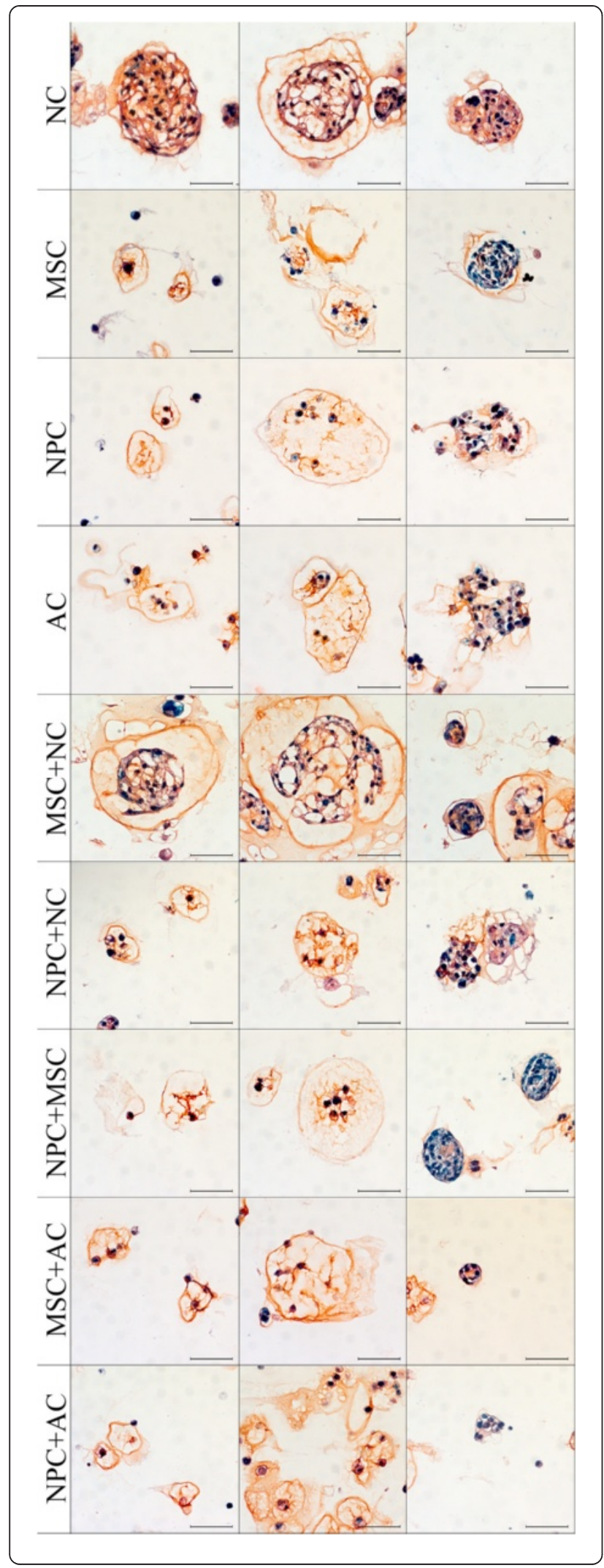

Figure 2 Extracellular matrix deposition. Histopathological slides of typical cell morphologies on day 28 of notochordal cells (NCs), mesenchymal stromal cells (MSCs), nucleus pulposus cells (NPCs), articular chondrocytes (ACs), MSC + NC, NPC + NC, NPC + MSC, $\mathrm{MSC}+\mathrm{AC}$, and $\mathrm{NPC}+\mathrm{AC}$. Prior to staining, alginate was removed with sodium citrate. Cell nuclei are stained blue (hematoxylin), proteoglycans are red (Safranin O) and collagen is green (Fast Green) (scale bar $=50 \mu \mathrm{m}$ ).

The brachyury gene expression was significantly higher in the NC and MSC + NC groups than in the other groups. KRT18 expression remained stable in all culture groups (Additional file 5). The ACAN and COL2A1 expression of NCs increased least of all groups over time, and the expression of ACAN and COL2A1 in the MSC + AC group increased significantly more with time than the $\mathrm{MSC}+\mathrm{NC}$ group (Figure 3D,E). Although COL1A1 expression in the NC group increased with time, coculture of MSC + NC showed a significantly greater decrease than the MSC + AC group (Figure 3F). Additional files 7 and 8 how in detail the statistical differences in gene expression levels between groups on day 0 and during culture.

\section{The regulatory effect of notochordal cells on nucleus} pulposus cells in coculture

The morphology of the NPCs on day 1 and 28 was similar to that of the ACs (Additional file 6). On day 28 , morphologies of the cocultured cells were the same as in monocultures. The DNA content of all culture groups remained unchanged over time (Figure 4A), and the amount of GAG/DNA increased significantly and similarly in all groups (Figure 4B, Additional file 3). Safranin O/Fast Green staining indicated that the cells in cocultures of $\mathrm{NPC}+\mathrm{NC}$ and NPC + AC deposited amounts of proteoglycans similar to the monocultures of the respective cell types (Figure 2).

Brachyury gene expression was significantly higher in the NC and NPC + NC groups at day 1 compared with the other groups, but expression increased significantly more in NCs alone than in coculture with NPCs (Figure 4C). KRT18 expression remained stable in all culture groups (Additional file 5). On day 1 in culture, NCs showed a significantly higher ACAN gene expression than NPCs. NPC + AC showed a significantly higher increase in $A C A N$ expression in comparison to $\mathrm{NPC}+\mathrm{NC}$. When we compared NPC, NC, NPC + NC, and NPC + AC groups, we observed no significant differences in changes in COL2A1 and COL1A1 expression (Figure $4 \mathrm{E}$ and $\mathrm{F}$ ). Additional files 7 and 8 show in detail the statistical differences on gene expression level between groups on day 0 and during culture. 

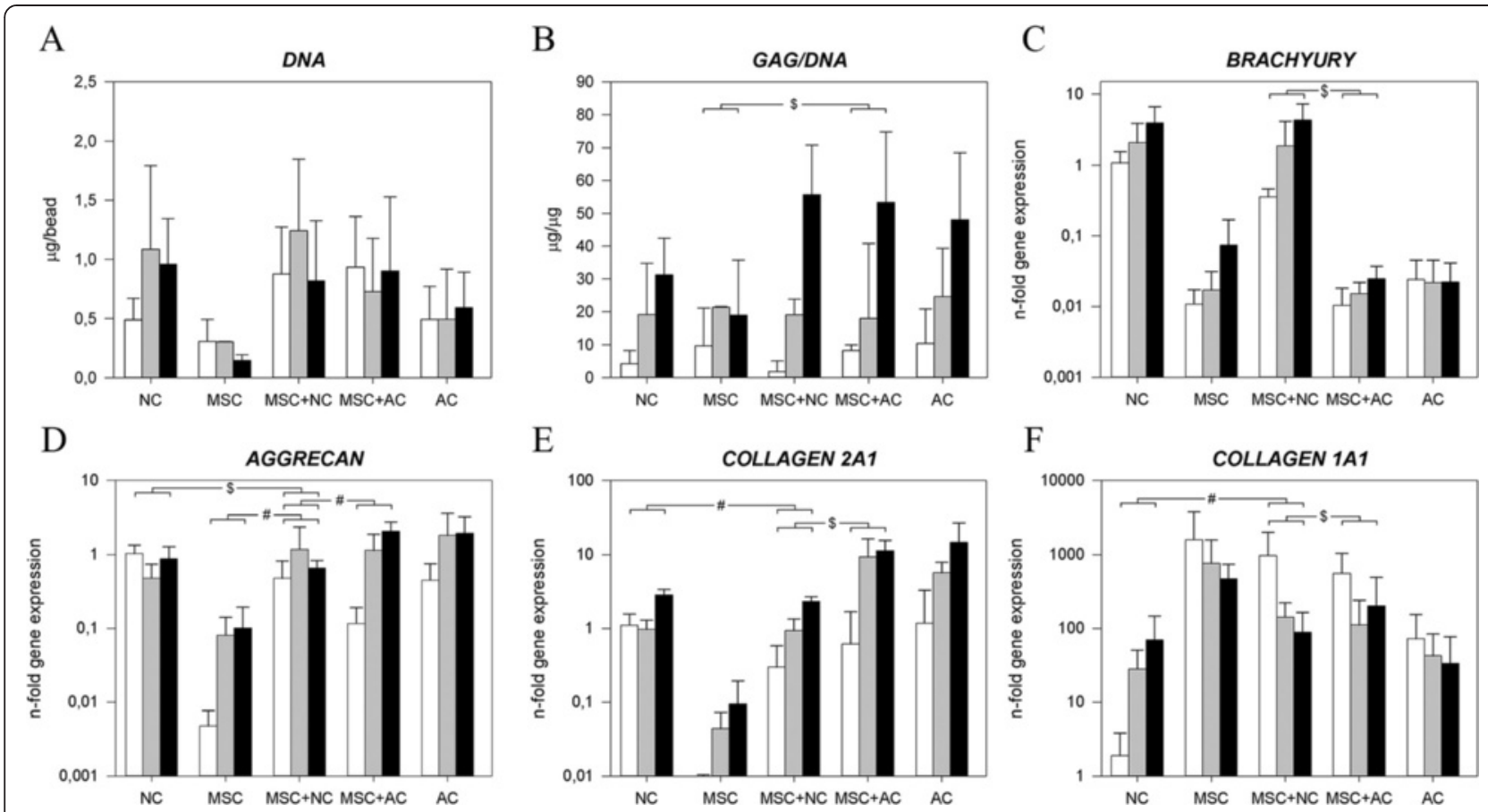

Figure 3 Notochordal cells and mesenchymal stromal cells in coculture (control: articular chondrocytes). Depiction of the (A) DNA content and (B) glycosaminoglycan (GAG) content normalized to DNA (GAG/DNA) and the relative gene expression of (C) notochordal cell (NC) marker brachyury; (D) aggrecan; (E) collagen, type II, a1 (collagen 2A1); and (F) collagen, type I, a1 (collagen 1A1). White bar = day 1, gray bar = day 15, black bar $=$ day 28. ${ }^{\$} P<0.05$, significant difference in the increase in GAG/DNA production/gene expression in time between groups. ${ }^{\#} P<0.001$, significant difference in the increase in gene expression in time between groups. Only relevant comparisons are displayed. MSC, Mesenchymal stromal (stem) cell; AC, Articular chondrocyte.

The regulatory effect of notochordal cells on nucleus pulposus cell vs. notochordal cells on mesenchymal stromal (stem) cells

When NPCs were combined with MSCs, the DNA content did not change significantly (Figure 5A). Unlike the high GAG/DNA of the MSC + NC group compared with monocultures, there was no synergistic effect on GAG production in the NPC $+\mathrm{NC}$ and NPC + MSC groups (Figure 5B, Additional file 3). These findings were confirmed by Safranin O/Fast Green staining.

Brachyury expression increased significantly more in the MSC + NC and MSC + NPC groups than in the $\mathrm{NPC}+\mathrm{NC}$ group (Figure $5 \mathrm{C}$ ). ACAN expression increased less in the NPC + NC group than in the MSC + $\mathrm{NC}$ group, but both increased less than in the NPC + MSC group (Figure 5D). COL2A1 expression was not significantly different between $\mathrm{MSC}+\mathrm{NC}$ and $\mathrm{NPC}+$ $\mathrm{NC}$ in time. Finally, COL1A1 decreased more in the $\mathrm{MSC}+\mathrm{NC}$ and MSC + NPC groups than for NPC + NC (Figure 5F). Additional files 7 and 8 show in detail the statistical differences in gene expression levels between groups on day 0 and during culture.

\section{Discussion}

The regenerative potential of notochordal cells

Cell-based regenerative strategies for the NP have used both NPCs and MSCs [32], but with limited success. NCs, involved in disc development, have been suggested to stimulate the regenerative capacity of the resident cells of the degenerated disc (NPCs), as well as the additionally introduced exogenous MSCs [33]. Previous short-term (3 days) NPC + NC coculture and culture of bovine NPCs in medium conditioned by canine NCs resulted in significantly increased proteoglycan metabolism [11]. The same was observed for human MSCs cultured in porcine NC-conditioned medium $[16,34]$. With an increased duration of NPC + NC coculture (14 days; bovine + porcine), still a slight, but significant increase in GAG/DNA compared with monoculture groups was observed [13]. However, in a long-term study (28 days), no effect of porcine NCs on the GAG production of a bovine NPC/MSC mix (ratio 1:1) was observed [35]. In the latter study, it was hypothesized that the change in phenotype of the NCs, as observed during the culture period, negatively affected the regenerative potential of these cells. 

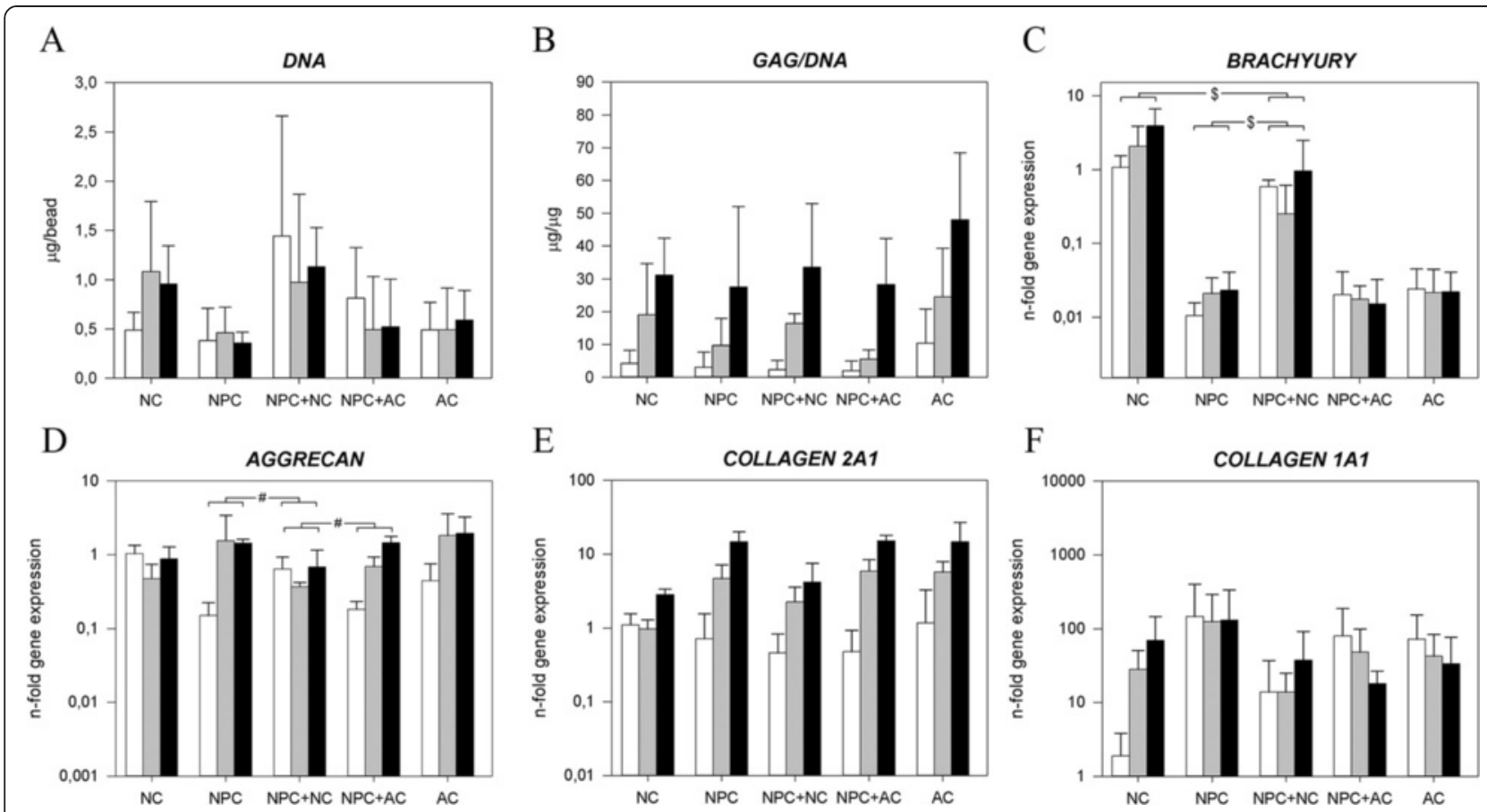

Figure 4 Notochordal cells (NCs) and nucleus pulposus cells (NPCs) in coculture (control: articular chondrocytes (ACs)). Depiction of the (A) DNA content and (B) glycosaminoglycan (GAG) content normalized to DNA (GAG/DNA) and the relative gene expression of (C) NC marker brachyury; (D) aggrecan; (E) collagen, type II, a1 (collagen 2A1); and (F) collagen, type I, a1 (collagen 1A1). White bar= day 1, gray bar = day 15, black bar = day $28 .{ }^{\$} P<0.05$, significant difference in the increase in gene expression in time between groups. ${ }^{\#} P<0.001$, significant difference in the increase in gene expression in time between groups. Only relevant comparisons are displayed.

In the present study, an in vitro coculture model was used to evaluate whether NCs could enhance the regenerative potential of NPCs and MSCs. ACs were employed as a control for NC-specific effects. Despite the small number of repeats, addition of NCs to MSCs in coculture did result in substantially more GAG/DNA compared with MSCs alone, but this was probably a result of increased GAG production by the NCs rather than of the NCs stimulating the MSCs. Similarly, when ACs were added to MSCs, more GAG/DNA was produced, but, again, this was through production by the ACs. When NCs, MSCs or ACs were added to NPCs, there was no stimulatory effect of coculture and the results were similar to those with NPCs alone. Thus, in this long-term, canine three-dimensional coculture system, there was no stimulation of NPCs or MSCs cocultured with other cells.

Loss of notochordal cell phenotype in long-term culture Despite the fact that in the present study monocultures of NCs maintained their brachyury and KRT18 expression at basal levels, the phenotype of the NCs changed during culture. Initially, all NCs contained large vacuoles and were organized in clusters, whereas at the end of the culture period three different populations of clustered NCs were observed: NCs with smaller vacuoles, fibroblast-like cells and small clusters of round cells. The total amount of cells in the NC cultures increased slightly in time, but the percentage of vacuolated NCs, which resemble the original population, decreased. Unlike the observation by Potier et al. that porcine NCs produced negligible amounts of proteoglycans during culture for 28 days [35], the canine cells in the NC group in the present study produced significant amounts of proteoglycans accompanied by significantly increased expression of COL2A1 and COL1A1 in time. This difference may be due to the chosen culture conditions or to the difference in species used. The assessment of additional markers for the NC phenotype, such as KRT8 and KRT19 [19,36], could have improved the insight into the phenotypical change in the present study.

NCs in clusters were (co)cultured in three-dimensions, in vitro, under physiological hypoxia and osmolarity, with the aim of maintaining their phenotype [24,37-40]. In previous studies, NCs were cultured in DMEM/F-12 supplemented with $8 \%$ to $15 \%$ FBS $[11,39]$. In the present study, the basal culture medium (high-glucose DMEM) used was chosen to support the chondrogenic potential of NPCs and MSCs [41]. Excess of glucose in this culture medium may have induced a change in NC phenotype. Adjustment of the medium composition may have been detrimental to the phenotype of the NCs, as 


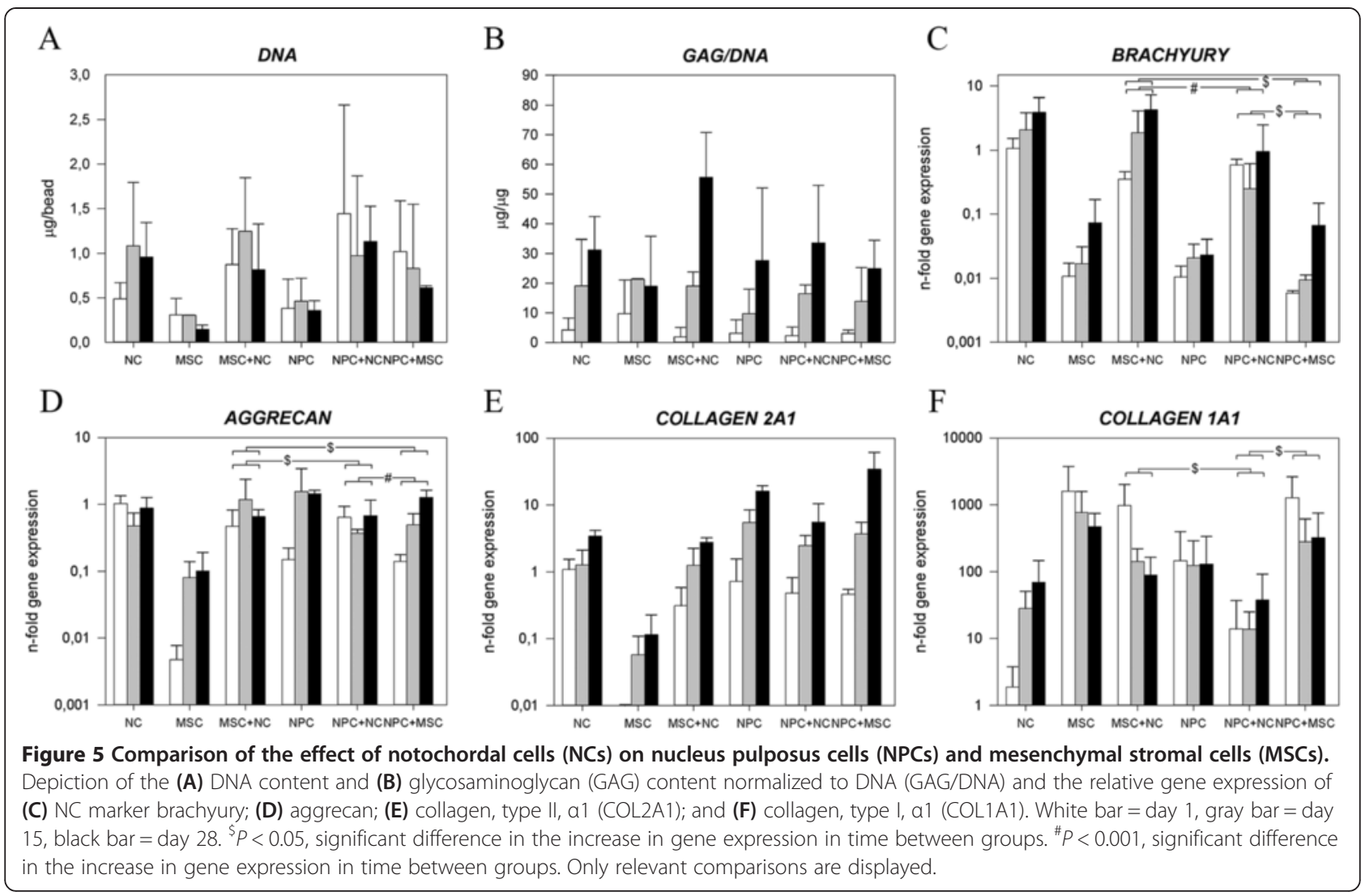

recent reports indicate that glucose deprivation, as well as high concentrations of glucose, results in decreased proliferation and increased apoptosis of NCs [37,42,43]. NCs cultured in either advanced DMEM/F-12 culture medium or minimum essential medium Eagle, alpha modification ( $\alpha$-MEM), have been shown to maintain their notochordal phenotype better than NCs cultured in simple DMEM/F-12 or DMEM $[24,44,45]$. It has been suggested that the presence of ascorbic acid in DMEM/ $\mathrm{F}-12$ and $\alpha-\mathrm{MEM}$ is the key factor for maintaining the NC phenotype [44].

Therefore, with the aim of preserving NC phenotype in long-term culture, it is recommended to culture NCs in clusters [40] in serum-free (unpublished results) $\alpha$ MEM at an osmolarity of $400 \mathrm{mOsm} / \mathrm{L}$ [24] under hypoxic conditions [39]. In addition, a physiological $\mathrm{pH}$ and compression could contribute to maintenance of the $\mathrm{NC}$ phenotype.

Trophic effects of mesenchymal stromal (stem) cells on notochordal cells

Whereas NCs were not able to induce a response of NPCs or MSCs in coculture, the MSCs appeared to regulate an increase in GAG production and $A C A N$ expression of the NCs. Only a few, single, chondrocyte-like cells could be identified in these cocultures on day 28, so it seems that only a small percentage of the MSCs (or NCs) differentiated to a chondrogenic phenotype. To the authors' knowledge, only one report on the effect of MSCs on NC behavior exists [46]. When MSCs were injected in murine NPs, with induced degeneration, the number of resident $\mathrm{NCs}$ and the differentiation of the resident NCs to NPC-like cells increased significantly compared with untreated degenerated NPs. Furthermore, half of the injected MSCs differentiated to NPClike cells after 4 weeks of culture. Altogether, this resulted in increased proteoglycan deposition by the entire cell population [46], based on the differentiation of MSCs toward NPC-like cells and their trophic effect on the resident cell population. In previous MSC + AC cocultures, of which more reports are available, similar observations were made: MSCs have mainly trophic effects on ACs in coculture, and, surprisingly, the majority of the MSCs disappears during culture. Only a minority of the MSCs differentiate to a chondrogenic phenotype, thereby contributing to the matrix production [47-49]. In this respect, the recently described niche of NP progenitor cells [50] may interact with the resident NCs and thereby play an important, but currently unidentified, role in the degenerative cascade of the IVD. 


\section{Study limitations}

The present study had some limitations. With regard to determining the fate of each cell type during 28 days in culture, the application of cell labeling (that is, with green fluorescent protein or long-term cytoplasmic staining) would have been valuable. Cell labeling would have shed a light on the final cell ratio, possible changes in cell morphology and cells secreting proteoglycans. Second, cell densities in monoculture $\left(3 \times 10^{6}\right.$ cells $\left./ \mathrm{ml}\right)$ and coculture $\left(6 \times 10^{6}\right.$ cells $\left./ \mathrm{ml}\right)$ were chosen in the range of the in vivo cell concentration in the human NP (4 to $5 \times 10^{6}$ cells $/ \mathrm{ml}$ ) [51]. However, the cell densities in the coculture groups were double those of the monoculture groups, with the aim of observing the additive effect of the supposedly regenerative NCs and control cell type ACs on MSCs and NPCs. However, this discrepancy in cell density may have affected the paracrine signaling and metabolism of the cells [52].

\section{Future studies}

We propose that $\mathrm{NC}$-conditioned medium, produced in culture conditions that support the NC phenotype, is a better alternative for evaluating the regenerative effect of NCs on NPC or MSCs, as it overcomes the drawbacks of loss of NC phenotype and bidirectional intercellular communication in culture. NC-conditioned medium has been shown to increase anabolic gene expression by NPCs [11,53] or MSCs [16,33] and to inhibit apoptosis of NCs [45]. Furthermore, challenging NPCs or MSCs in in vitro tissue models in the presence of $\mathrm{NC}$-conditioned medium would give further insight into in vivo regenerative processes.

\section{Conclusions}

Direct addition of canine NCs did not have a regenerative effect on canine MSCs or NPCs in the present study design. The lack of a regenerative response may be due to a change in phenotype of the NCs under culture conditions that support the chondrogenic potential of MSCs and NPCs. This may be avoided in future studies by using NCconditioned medium. These results have implications for strategies using NCs and MSCs for IVD regeneration.

\section{Additional files}

Additional file 1: List of primers for gene expression analysis.

Additional file 2: Cell viability. Cell viability on day 1 of notochordal cells (NCs), mesenchymal stromal cells (MSC), nucleus pulposus cells (NPCs), articular chondrocytes (ACs), MSC + NC, NPC + NC, NPC + MSC, $\mathrm{MSC}+\mathrm{AC}$ and NPC + AC. Cytoplasm of living cells was stained with calcein-AM (green fluorescence), and DNA of dead cells was stained with propidium iodide (red fluorescence) (scale bar $=200 \mu \mathrm{m}$ ).

Additional file 3: $P$-values of changes in DNA and GAG/DNA content during culture. NS: not significant with $P>0.1$.
Additional file 4: $P$-values of changes in gene expression within monoculture groups. NS: not significant with $P>0.1$.

Additional file 5: Cytokeratin 18 expression. Depiction of the relative gene expression of cytokeratin 18 in the following comparisons. (A) The effect of notochordal cells (NCs) on mesenchymal stromal cells (MSCs). (B) The effect of NCs on nucleus pulposus cells (NPCs). (C) The effect of NCs on NPCs vs. MSCs. White bar = day 1, gray bar = day 15, black bar $=$ day 28.

Additional file 6: Mesenchymal stromal cells (MSCs), nucleus pulposus cells (NPCs) and articular chondrocytes (ACs) in culture. Histopathological slides (H\&E) of (A) AC on day 1 (similar to NPC and MSC on day 1). (B) through (D) Day $28 \mathrm{H \& E}$ staining of (B) MSCs, (C) NPCs and (D) ACs (scale bar $=100 \mu \mathrm{m})$. The (E) DNA content, $(\mathbf{F})$ glycosaminoglycan content normalized to DNA (GAG/DNA) and gene expression of (G) aggrecan, $\mathbf{( H )}$ collagen type 2, a1, and (I) collagen type 1, a1. White bar $=$ day 1 , gray bar $=$ day 15 , black bar $=$ day $28 .{ }^{*} P<0.01$, significantly different from day 1.

Additional file 7: $P$-values of differences in gene expression between groups on day 0 . NS: not significant with $P>0.1$.

Additional file 8: $P$-values of differences in change in gene expression between groups during culture. NS: not significant with $P>0.1$.

\section{Abbreviations}

AC: Articular chondrocyte; ACAN: Aggrecan; AF: Annulus fibrosus; a-MEM: Minimum essential medium Eagle, alpha modification; CD: Chondrodystrophic; COL1A1: Collagen, type I, a1; COL2A1: Collagen, type II, a 1; DMEM: Dulbecco's modified Eagle's medium; FBS: Fetal bovine serum; GAG: Glycosaminoglycan; GAPDH: Glyceraldehyde 3-phosphate dehydrogenase; H\&E: Hematoxylin and eosin; HYP: Hydroxyproline; IVD: Intervertebral disc; KRT18: Cytokeratin 18; MSC: Mesenchymal stromal (stem) cell; NC: Notochordal cell; NCD: Nonchondrodystrophic; NP: Nucleus pulposus; NPC: Nucleus pulposus cell; PI: Propidium iodide; P/S: Penicillin-streptomycin; qPCR: Quantitative RT-PCR; RPS19: Ribosomal protein S19; SD: Standard deviation; TBP: TATA-box-binding protein.

\section{Competing interests}

The authors declare that they have no competing interests.

\section{Authors' contributions}

EP, Kl, BM and MT conceived the study and participated in its design and coordination. IA, SS, FR, BM and MT carried out the experiments. IA, EP, LS, MT and SS processed the data. LS performed the statistical analysis. IA, EP, $\mathrm{LS}, \mathrm{BM}, \mathrm{KI}$ and MT performed the final data analysis and interpretation. IA drafted the manuscript, which was edited by all authors. All authors read and approved the final manuscript.

\section{Acknowledgements}

The authors gratefully acknowledge Frank Riemers for technical assistance in qPCR, Nicole Willems for assistance in tissue sampling, Shradda Thakkar for assistance in sample sectioning and Marina van Doeselaar for assistance in histological staining. This study was funded by AOSpine International (grant SRN-2011-11). The funding source did not play any role in study design; collection, analysis or interpretation of data; manuscript writing; or the decision to submit the manuscript for publication. Marianna Tryfonidou was supported by the Dutch Arthritis Foundation (LLP22).

\section{Author details}

${ }^{1}$ Department of Biomedical Engineering, Eindhoven University of Technology, P.O. Box 513, 5600 MB Eindhoven, The Netherlands. ${ }^{2}$ Department of Clinical Sciences of Companion Animals, Faculty of Veterinary Medicine, Utrecht University, PO Box 80.154, NL-3508 TD Utrecht, The Netherlands. ${ }^{3}$ Clinic for Small Animal Surgery, Vetsuisse Faculty, Zurich University, Winterthurerstrasse 260, CH-8057 Zurich, Switzerland. ${ }^{4}$ Laboratoire de Bioingénierie et Biomécanique Ostéo-Articulaire (B2OA), UMR CNRS 7052, Université Denis Diderot Paris 7, Sorbonne Paris Cité, 690 Paris, France. ${ }^{5}$ Department of Orthopedics, University Medical Center Utrecht, P.O. Box 85500, HP G05.228, 3508 GA, Utrecht, The Netherlands. 
Received: 3 June 2014 Accepted: 19 February 2015

\section{Published online: 14 March 2015}

\section{References}

1. Buchbinder R, Blyth FM, March LM, Brooks P, Woolf AD, Hoy DG. Placing the global burden of low back pain in context. Best Pract Res Clin Rheumatol. 2013;27:575-89.

2. Kandel R, Roberts $S$, Urban JPG. Tissue engineering and the intervertebral disc: the challenges. Eur Spine J. 2008;17(Suppl 4):480-91.

3. Anderson DG, Tannoury C. Molecular pathogenic factors in symptomatic disc degeneration. Spine J. 2005;5(6 Suppl):260S-266S.

4. Walker MH, Anderson DG. Molecular basis of intervertebral disc degeneration. Spine J. 2004:4(6 Suppl):158S-166S.

5. Zhao CQ, Wang LM, Jiang LS, Dai LY. The cell biology of intervertebral disc aging and degeneration. Ageing Res Rev. 2007;6:247-61.

6. Bergknut N, Rutges JPHJ, Kranenburg HJC, Smolders LA, Hagman R, Smidt $\mathrm{HJ}$, et al. The dog as an animal model for intervertebral disc degeneration? Spine (Phila Pa 1976). 2012;37:351-8.

7. Bergknut N, Smolders LA, Grinwis GCM, Hagman R, Lagerstedt AS Hazewinkel HAW, et al. Intervertebral disc degeneration in the dog. Part 1: anatomy and physiology of the intervertebral disc and characteristics of intervertebral disc degeneration. Vet J. 2013;195:282-91.

8. Smolders LA, Bergknut N, Grinwis GCM, Hagman R, Lagerstedt AS, Hazewinkel HAW, et al. Intervertebral disc degeneration in the dog. Part 2: chondrodystrophic and non-chondrodystrophic breeds. Vet J. 2013;195:292-9.

9. Hunter CJ, Matyas JR, Duncan NA. Cytomorphology of notochordal and chondrocytic cells from the nucleus pulposus: a species comparison. J Anat. 2004;205:357-62

10. Cappello R, Bird JLE, Pfeiffer D, Bayliss MT, Dudhia J. Notochordal cell produce and assemble extracellular matrix in a distinct manner, which may be responsible for the maintenance of healthy nucleus pulposus. Spine (Phila Pa 1976). 2006;31:873-83.

11. Aguiar DJ, Johnson SL, Oegema TR. Notochordal cells interact with nucleus pulposus cells: regulation of proteoglycan synthesis. Exp Cell Res. 1999:246:129-37.

12. Abbott RD, Purmessur D, Monsey RD, latridis JC. Regenerative potential of TGFB3 + Dex and notochordal cell conditioned media on degenerated human intervertebral disc cells. J Orthop Res. 2012;30:482-8.

13. Gantenbein-Ritter $B_{1}$ Chan SCW. The evolutionary importance of cell ratio between notochordal and nucleus pulposus cells: an experimental 3-D coculture study. Eur Spine J. 2012;21(Suppl 6):S819-25.

14. Stoyanov JV, Gantenbein-Ritter B, Bertolo A, Aebli N, Baur M, Alini M, et al. Role of hypoxia and growth and differentiation factor-5 on differentiation of human mesenchymal stem cells towards intervertebral nucleus pulposus-like cells. Eur Cell Mater. 2011;21:533-47.

15. Henriksson HB, Svanvik T, Jonsson M, Hagman M, Horn M, Lindahl A, et al. Transplantation of human mesenchymal stems cells into intervertebral discs in a xenogeneic porcine model. Spine (Phila Pa 1976). 2009:34:141-8.

16. Korecki CL, Taboas JM, Tuan RS, latridis JC. Notochordal cell conditioned medium stimulates mesenchymal stem cell differentiation toward a young nucleus pulposus phenotype. Stem Cell Res Ther. 2010;1:18.

17. Chan SCW, Gantenbein-Ritter B, Leung VYL, Chan D, Cheung KMC, Ito K. Cryopreserved intervertebral disc with injected bone marrow-derived stromal cells: a feasibility study using organ culture. Spine J. 2010;10:486-96.

18. Hiyama A, Mochida J, Iwashina T, Omi H, Watanabe T, Serigano K, et al. Transplantation of mesenchymal stem cells in a canine disc degeneration model. J Orthop Res. 2008;26:589-600.

19. Minogue BM, Richardson SM, Zeef LA, Freemont AJ, Hoyland JA. Transcriptional profiling of bovine intervertebral disc cells: implications for identification of normal and degenerate human intervertebral disc cell phenotypes. Arthritis Res Ther. 2010;12:R22.

20. Smolders LA, Meij BP, Riemers FM, Licht R, Wubbolts R, Heuvel D, et al. Canonical Wnt signaling in the notochordal cell is upregulated in early intervertebral disk degeneration. J Orthop Res. 2012;30:950-7.

21. Tryfonidou MA, Schumann S, Armeanu S, Harichandan A, Sivasubramaniyan K, Mollenhauer J, et al. Update on canine MSC markers. Cytometry A. 2014:85:379-81.

22. Guo JF, Jourdian GW, MacCallum DK. Culture and growth characteristics of chondrocytes encapsulated in alginate beads. Connect Tissue Res. 1989;19:277-97.
23. van Dijk BGM, Potier E, Ito K. Long-term culture of bovine nucleus pulposus explants in a native environment. Spine J. 2013;13:454-63.

24. Spillekom S, Smolders LA, Grinwis GCM, Arkesteijn ITM, Ito K, Meij BP, et al. Increased osmolarity and cell clustering preserve canine notochordal cell phenotype in culture. Tissue Eng Part C Methods. 2014;20:652-62.

25. Cesarone CF, Bolognesi C, Santi L. Improved microfluorometric DNA determination in biological material using 33258 Hoechst. Anal Biochem. 1979;100:188-97.

26. Farndale RW, Buttle DJ, Barrett AJ. Improved quantitation and discrimination of sulphated glycosaminoglycans by use of dimethylmethylene blue. Biochim Biophys Acta. 1986;883:173-7.

27. Huszar G, Maiocco J, Naftolin F. Monitoring of collagen and collagen fragments in chromatography of protein mixtures. Anal Biochem. 1980;105:424-9.

28. Livak KJ, Schmittgen TD. Analysis of relative gene expression data using real-time quantitative PCR and the $2 T^{-\Delta \Delta C}$ method. Methods. 2001;25:402-8.

29. R Core Team. R: a language and environment for statistical computing 2014. http://www.r-project.org/. Accessed 23 Mar 2015.

30. Pinheiro J, Bates D, DebRoy S, Sarkar D, EISPACK authors, R Core Team. nlme: linear and nonlinear mixed effects models. R package version 3.1-120. http://cran.r-project.org/web/packages/nlme/index.html. Accessed 23 Mar 2015.

31. Benjamini $Y$, Hochberg $Y$. Controlling the false discovery rate: a practical and powerful approach to multiple testing. J R Stat Soc Series B Stat Methodol. 1995;57:289-300

32. Hohaus C, Ganey TM, Minkus Y, Meisel HJ. Cell transplantation in lumbar spine disc degeneration disease. Eur Spine J. 2008;17 Suppl 4:492-503.

33. Purmessur D, Cornejo MC, Cho SK, Hecht AC, latridis JC. Notochorda cell-derived therapeutic strategies for discogenic back pain. Global Spine J. 2013:3:201-18

34. Purmessur D, Schek RM, Abbott RD, Ballif BA, Godburn KE, latridis JC. Notochordal conditioned media from tissue increases proteoglycan accumulation and promotes a healthy nucleus pulposus phenotype in human mesenchymal stem cells. Arthritis Res Ther. 2011;13:R81.

35. Potier E, Ito K. Using notochordal cells of developmental origin to stimulate nucleus pulposus cells and bone marrow stromal cells for intervertebral disc regeneration. Eur Spine J. 2014;23:679-88.

36. Weiler C, Nerlich AG, Schaaf R, Bachmeier BE, Wuertz K, Boos N. Immunohistochemical identification of notochordal markers in cells in the aging human lumbar intervertebral disc. Eur Spine J. 2010;19:1761-70.

37. Guehring T, Wilde G, Sumner M, Grünhagen T, Karney GB, Tirlapur UK, et al. Notochordal intervertebral disc cells: sensitivity to nutrient deprivation. Arthritis Rheum. 2009;60:1026-34

38. Mwale F, Ciobanu I, Giannitsios D, Roughley P, Steffen T, Antoniou J. Effect of oxygen levels on proteoglycan synthesis by intervertebral disc cells. Spine (Phila Pa 1976). 2011;36:E131-8.

39. Erwin WM, Las Heras F, Islam D, Fehlings MG, Inman RD. The regenerative capacity of the notochordal cell: tissue constructs generated in vitro under hypoxic conditions. J Neurosurg Spine. 2009;10:513-21.

40. Hunter CJ, Matyas JR, Duncan NA. The functional significance of cell clusters in the notochordal nucleus pulposus: survival and signaling in the canine intervertebral disc. Spine (Phila Pa 1976). 2004:29:1099-104

41. Phitak T, Pothacharoen $P$, Kongtawelert $P$. Comparison of glucose derivatives effects on cartilage degradation. BMC Musculoskelet Disord. 2010;11:162.

42. Park EY, Park JB. Dose- and time-dependent effect of high glucose concentration on viability of notochordal cells and expression of matrix degrading and fibrotic enzymes. Int Orthop. 2013;37:1179-86.

43. Won HY, Park JB, Park EY, Riew KD. Effect of hyperglycemia on apoptosis of notochordal cells and intervertebral disc degeneration in diabetic rats. J Neurosurg Spine. 2009;11:741-8.

44. Rastogi A, Thakore P, Leung A, Benavides M, Machado M, Morschauser MA, et al. Environmental regulation of notochordal gene expression in nucleus pulposus cells. J Cell Physiol. 2009;220:698-705

45. Erwin WM, Islam D, Inman RD, Fehlings MG, Tsui FWL. Notochordal cells protect nucleus pulposus cells from degradation and apoptosis: implications for the mechanisms of intervertebral disc degeneration. Arthritis Res Ther. 2011;13:R215.

46. Yang F, Leung VYL, Luk KDK, Chan D, Cheung KMC. Mesenchymal stem cells arrest intervertebral disc degeneration through chondrocytic differentiation and stimulation of endogenous cells. Mol Ther. 2009:17:1959-66. 
47. Wang M, Rahnama R, Cheng T, Grotkopp E, Jacobs L, Limburg S, et al. Trophic stimulation of articular chondrocytes by late-passage mesenchymal stem cells in coculture. J Orthop Res. 2013;31:1936-42.

48. Wu L, Leijten JCH, Georgi N, Post JN, van Blitterswijk CA, Karperien M. Trophic effects of mesenchymal stem cells increase chondrocyte proliferation and matrix formation. Tissue Eng Part A. 2011;17:1425-36.

49. Acharya C, Adesida A, Zajac P, Mumme M, Riesle J, Martin I, et al. Enhanced chondrocyte proliferation and mesenchymal stromal cells chondrogenesis in coculture pellets mediate improved cartilage formation. J Cell Physiol. 2012;227:88-97.

50. Erwin WM, Islam D, Eftekarpour E, Inman RD, Karim MZ, Fehlings MG. Intervertebral disc-derived stem cells: implications for regenerative medicine and neural repair. Spine (Phila Pa 1976). 2013;38:211-6.

51. Kobayashi S, Meir A, Urban J. Effect of cell density on the rate of glycosaminoglycan accumulation by disc and cartilage cells in vitro. J Orthop Res. 2008;26:493-503.

52. Stephan $\mathrm{S}$, Johnson WE, Roberts $\mathrm{S}$. The influence of nutrient supply and cell density on the growth and survival of intervertebral disc cells in 3D culture. Eur Cell Mater. 2011;22:97-108.

53. Erwin WM, Inman RD. Notochord cells regulate intervertebral disc chondrocyte proteoglycan production and cell proliferation. Spine (Phila Pa 1976). 2006;31:1094-9.

\section{Submit your next manuscript to BioMed Central and take full advantage of:}

- Convenient online submission

- Thorough peer review

- No space constraints or color figure charges

- Immediate publication on acceptance

- Inclusion in PubMed, CAS, Scopus and Google Scholar

- Research which is freely available for redistribution 